\title{
High pemetrexed sensitivity of docetaxel-resistant A549 cells is mediated by TP53 status and downregulated thymidylate synthase
}

\author{
WEI-TING KUO ${ }^{1,2^{*}}$, DOM-GENE TU ${ }^{3-5^{*}}$, LING-YEN CHIU ${ }^{6}$, GWO-TARNG SHEU ${ }^{6}$ and MING-FANG WU ${ }^{7,8}$ \\ ${ }^{1}$ Institute of Clinical Medicine, National Yang-Ming University, Taipei 112; ${ }^{2}$ Department of Surgery, \\ Chia-Yi Christian Hospital; ${ }^{3}$ Department of Nuclear Medicine, Ditmanson Medical Foundation, \\ Chia-Yi Christian Hospital, Chiayi City 60002; ${ }^{4}$ Department of Food Science and Technology, \\ Chia Nan University of Pharmacy and Science, Tainan City $717 ;{ }^{5}$ College of Health Sciences, \\ Chang Jung Christian University, Tainan City $711 ;{ }^{6}$ Institute of Medicine, ${ }^{7}$ School of Medicine, \\ Chung Shan Medical University and ${ }^{8}$ Divisions of Medical Oncology and Chest Medicine, \\ Chung Shan Medical University Hospital, Taichung City 402, Taiwan, R.O.C.
}

Received February 22, 2017; Accepted August 2, 2017

DOI: $10.3892 / o r .2017 .5951$

\begin{abstract}
The chemoresistance of non-small cell lung cancer (NSCLC) that occurs in docetaxel (DOC) chemotherapy substantially decreases the survival of patients. To overcome DOC-induced chemoresistance, we established DOC-selected A549 lung cancer sublines (A549/D16 and A549/D32) and revealed that both sublines were cross-resistant to vincristine (VCR) and doxorubicin (DXR). Notably, both sublines were more sensitive to pemetrexed (PEM) than parental cells according to MTT and clonogenic assays. The expression levels of thymidylate synthase (TS) and $\gamma$-glutamyl hydrolase (GGH) were downregulated in DOC-resistant sublines. When exogenous TS was overexpressed in A549/D16 cells, PEM sensitivity was significantly decreased, however it was not decreased by overexpression of exogenous GGH. PEM treatment induced more apoptotic sub-G1 cells in both DOC-resistant sublines and in the in vivo PEM sensitivities of A549/D16 cells. These findings were further confirmed by a xenografted tumor model. To unmask the mediator of TS downregulation, we investigated human lung cancer cell lines that have various TP53 statuses using DOC treatment. The level of TS protein was significantly decreased in wild-type TP53-containing cells with DOC treatment; TS expression levels were not affected in mutant-TP53 and TP53-null cells under the same conditions.
\end{abstract}

Correspondence to: Dr Ming-Fang Wu, Divisions of Medical Oncology and Chest Medicine, Chung Shan Medical University Hospital, 110 Section 1, Jianguo N. Road, Taichung City 402, Taiwan, R.O.C.

E-mail:mfwu0111@gmail.com

*Contributed equally

Key words: docetaxel, pemetrexed, thymidylate synthase, TP53, chemoresistance
Furthermore, when the expression of TP53 was inhibited in A549 cells, the expression level of TS was increased. Our data indicated that DOC activated wild-type TP53 and suppressed TS expression under continuous DOC exposure. Therefore, the expression of TS remained at low levels in DOC-resistant A549 cancer cells. Our data revealed that for lung cancer with DOC resistance and wild-type TP53 status, the administration of PEM as a second-line agent to overcome DOC-resistance may benefit patients.

\section{Introduction}

Lung cancer is the leading cause of death among males worldwide from all cancers (1), and non-small cell lung cancer (NSCLC) accounts for more than $85 \%$ of lung cancer cases; of which adenocarcinoma (40\%) is the most common subtype, followed by squamous cell $(25 \%)$ and large cell carcinoma (10\%) (2). Chemotherapy is the most common treatment for advanced-stage lung cancer patients. Such therapy is generally performed with platinum-based chemotherapy. However, this treatment provides only a modest benefit to survival. Unsatisfactorily, the overall 5-year survival rate for all stages of NSCLC is only $17 \%$ (3). The unavoidable development of drug resistance is the most important cause of treatment failure in patients subjected to chemotherapy. When tumors become resistant to chemotherapeutic drugs, they commonly develop cross-resistance to other anticancer drugs. Therefore, choosing an ideal non-cross-resistant drug that improves drug responsiveness and increases the overall survival rate has become a critical issue in cancer management.

The taxanes, paclitaxel (PAX) and docetaxel (DOC), are microtubule-stabilizing agents that function primarily by interfering with spindle microtubule dynamics causing cell cycle arrest and apoptosis (4). The chemical statuses of the two taxanes are almost identical. DOC is regarded as a second-generation taxane. The effects of DOC are correlated with mitotic arrest and cellular toxicity (5). Currently, DOC is 
indicated for first-line therapy in combination with a platinum compound and as monotherapy in patients with NSCLC who experience failure during a platinum-based regimen.

P-glycoprotein (P-gp) is the product of the MDR1 gene, which is also recognized as ABCB1. P-gp is a pump that presents in normal tissues such as the gastrointestinal tract and brain to prevent the accumulation of toxic substances (6). Overexpression of P-gp is thought to be one of the most common mechanisms underlying resistance to taxanes in cancer models (7).

Pemetrexed (PEM; LY231514) is a novel antifolate drug that has been approved for first-line treatment of patients with advanced non-squamous NSCLC in combination with cisplatin. PEM is also used as a single agent for relapsed or chemotherapy refractory NSCLC after platinum-containing chemotherapy (8). PEM is a unique folate antagonist that inhibits thymidylate synthase (TS), dihydrofolate reductase (DHFR) and the purine synthetic enzyme glycinamide ribonucleotide formyltransferase (GARFT) as multitargeted antifolate $(9,10)$.

Two adenocarcinoma cell lines (PC-9 and A549) with PEM resistance were established and recently analyzed. TS and DHFR were significantly increased in the 4 PEM-resistant A549 sublines (11). Data obtained from patients have also suggested that TS expression, rather than DHFR, may be an important predictive factor of the treatment efficacy of PEM in NSCLC (12). Furthermore, it has been concluded by meta-analysis (13) that better responses usually appeared in patients with a lower expression of TS with a significant association between TS expression and outcomes of PEM-based chemotherapy for NSCLC. Therefore, it is believed that upregulation of TS gene expression may play an important role in PEM resistance (14) and downregulation of TS may increase PEM response.

Previously, we reported that DOC-selected A549 sublines (A549/D16 and A549/D32) expressed high levels of P-gp and were cross-resistant to VCR and DXR (15). We further defined the characteristics of these sublines with antifolate drugs (PEM and MTX) in the present study. We found that only PEM overcame DOC resistance and that TS in the de novo nucleic acid synthesis pathway may be downregulated by wild-type TP53 in DOC-selected A549 sublines. We further demonstrated that TS was downregulated by DOC-activated wild-type TP53 in two human lung cancer cell lines (A549 and H460), however it was not downregulated in cells with mutated TP53 and null-TP53. Therefore, according to our data, the application sequence of DOC followed by PEM in patients with wild-type TP53 status is suggested. This approach may lead to better cancer control in NSCLC.

\section{Materials and methods}

Drugs and chemicals. PEM was provided by Eli Lilly Corporation (Indianapolis, IN, USA). Doxorubicin (DXR; 44584), methotrexate (MTX) hydrate (M8407) and vincristine sulfate salt (VCR; V8879) were purchased from Sigma-Aldrich (St. Louis, MO, USA). The drugs were dissolved in $\mathrm{dd}_{2} \mathrm{O}$ (PEM, DXR and VCR), MTX was dissolved in 10\% dimethyl sulfoxide (DMSO) stored at $-20^{\circ} \mathrm{C}$, and was diluted in culture medium immediately before use. DOC (10 mg) was obtained from Aventis Pharmaceuticals Inc. (Bridgewater, NJ, USA) and was dissolved in $11.2 \mathrm{ml}$ DMSO and $20 \mathrm{ml}$ absolute ethanol as a $375 \mu \mathrm{M}$ stock.
Lung cancer cell lines. Human A549, H460 and H1355 cells were cultured at $37^{\circ} \mathrm{C}$ in Dulbecco's modified Eagle's medium (DMEM) supplemented with $10 \%$ fetal bovine serum (FBS), $1 \%$ NEAA, $1 \%$ sodium pyruvate, $1 \%$ L-glutamine, $100 \mathrm{IU} / \mathrm{ml}$ penicillin and $100 \mathrm{mg} / \mathrm{ml}$ streptomycin. H1299 and CL1-0 cells were cultured in RPMI-1640 medium. The DOC resistant sublines were established from parental cells in a stepwise manner by exposure to increasing concentrations of DOC as previously described (15). The DOC-resistant sublines maintained at 16 and $32 \mathrm{nM}$ of DOC are denoted as A549/D16 and A549/D32, respectively.

Cytotoxicity assay (MTT assay). Chemosensitivity to PEM was determined using an MTT assay. The detailed steps of MTT assay have been previously described (15). The cells were exposed to various concentrations of PEM in fresh medium for $96 \mathrm{~h}$. Mean values were calculated from 3 independent experiments.

Protein extraction and western blot analysis. Protein extracts were prepared as previously described (15). Proteins (10-30 $\mu \mathrm{g}$ ) transferred onto polyvinylidene fluoride (PVDF) membranes were reacted with polyclonal anti-TS (Santa Cruz Biotechnology, Inc., Santa Cruz, CA, USA), anti-DHFR, FPGS, GGH and TK1 (GeneTex, Irvine, CA, USA), anti- $\beta$-actin (NeoMarker, Fremont, CA, USA) and anti-TP53 (Dako, Carpinteria, CA, USA) separately, followed by conjugation of anti-rabbit (Santa Cruz Biotechnology, Inc.) or anti-mouse (Calbiochem, La Jolla, CA, USA) IgG to horseradish peroxidase. A chemiluminescence detection kit (ECL; GE Healthcare Bioscience, Amersham Place, UK) was used to determine the levels of protein expression. The Image $\mathbf{J}$ quantity software was downloaded from the National Institutes of Health to measure the intensity of each blot.

Clonogenic cell survival assay. Cells were seeded in 6-well plates ( 150 cells/plate). After $24 \mathrm{~h}$ of incubation, the cells were treated with respective doses of DOC (48 h) or PEM (96 h) and eventually cultured for 10 days. The colonies formed were fixed with ice-cold methanol for $30 \mathrm{~min}$ and then stained with $20 \%$ Giemsa. Survival fractions were calculated by normalization to the appropriate control groups.

Cell cycle assay by flow cytometry. For the cell cycle analysis, cells $\left(3 \times 10^{5}\right)$ were seeded in $10-\mathrm{cm}$ plates and incubated for $24 \mathrm{~h}$. The cells were exposed to PEM (0-110 nM) with fresh medium for $96 \mathrm{~h}$ at $37^{\circ} \mathrm{C}$. Cells were trypsinized and washed, then resuspended in $75 \%$ ice-cold ethanol $(75 \%)$ overnight at $4^{\circ} \mathrm{C}$. This was followed by washing with $1 \mathrm{X}$ PBS and centrifugation; the cell pellets were gently dispersed with $300 \mu \mathrm{l}$ of $1 \mathrm{X}$ PBS with $30 \mu \mathrm{l}$ of RNase A $(10 \mathrm{mg} / \mathrm{ml})$ for $30 \mathrm{~min}$. Propidium iodide (PI) $(10 \mu \mathrm{l} ; 500 \mu \mathrm{g} / \mathrm{ml})$ and $300 \mu \mathrm{l}$ of 1X PBS were added followed by another $30 \mathrm{~min}$ incubation at room temperature. Subsequently the cells were filtered by a nylon mesh $(40 \mu \mathrm{m})$, and then analyzed with a FACSCalibur flow cytometer (BD Biosciences, San Jose, CA, USA).

TS, GGH cloning and expression. To clone the human TS ORF for expression, the total RNA of H1299 cells was reverse-transcribed into cDNA by the MultiScribe reverse 
Table I. Characterization of drug sensitivities with DOC-resistant A549 sublines by MTT assay.

\begin{tabular}{lccc}
\hline & \multicolumn{3}{c}{$\mathrm{IC}_{50} \pm \mathrm{SD}^{\mathrm{a}}(\mathrm{nmol} / \mathrm{l})$} \\
\cline { 2 - 4 } Drug $(\mathrm{nM})$ & $\mathrm{A} 549$ & $\mathrm{~A} 549 / \mathrm{D} 16$ & $\mathrm{~A} 549 / \mathrm{D} 32$ \\
\hline Docetaxel & $7.6 \pm 1.8(1.0)$ & $829.2 \pm 28.6(109.1)$ & $2177.9 \pm 21.2(286.5)$ \\
Vincristine & $20.0 \pm 2.2(1.0)$ & $690.1 \pm 25.7(34.5)$ & $811.0 \pm 27.4(40.5)$ \\
Doxorubicin & $316.7 \pm 14.0(1.0)$ & $3,468.3 \pm 29.1(10.9)$ & $8,028.0 \pm 24.1(25.3)$ \\
Pemetrexed & $1304.7 \pm 94.7(1.0)$ & $187.7 \pm 2.5(0.1)$ & $120.3 \pm 8.6(0.1)$ \\
Methotrexate & $48.7 \pm 0.6(1.0)$ & $87.0 \pm 1.7(1.8)$ & $95.0 \pm 1.0(2.0)$ \\
\hline
\end{tabular}

${ }^{\mathrm{a}}$ Data are expressed as the mean $\pm \mathrm{SD}$ of at least 3 independent experiments carried out in triplicate. DOC, docetaxel.

transcription system (Applied Biosystems, Foster City, CA, USA). The forward primer (5'-TCTCGCTAGCATGCCTGTG GCCGGCTCGGA) and the reverse primer (5'-TCTCGTTTA AACCTAAACAGCCATTTCCATTT) were applied to amplify the ORF of TS by PCR. The obtained PCR product was digested with $N h e I$ and $P m e I$, and then purified. The ORF fragment was ligated into the lentiviral vector pLAS3w.Ppuro, which was provided by the National RNAi Core Facility Platform (Academia Sinica, Taiwan). The forward primer (5'-TCTCGCTAGCATGGCCAGTCCGGGCTGCCT) and the reverse primer (5'-TCTCCCTGCAGGTCAATCAAATA TGTAACATT) for GGH were also used to clone the gene into the same vector, followed by NheI and $S b f \mathrm{I}$ digestion and ligation. A549 and A549/D16 cells that were seeded in 6-cm plates were transduced with the lentivirus carrying TS, GGH or empty vector as control to express TS or GGH for $48 \mathrm{~h}$. The transduced cells were harvested and seeded into 24 -well plates for MTT assay as previously described.

Mice xenograft models. Experimental procedures and handling were conducted in accordance with the international guidelines for laboratory animals and all efforts were made to minimize suffering. The present study was approved by the Chung Shan Medical University Animal Care Committee (permit no. 1238). To establish A549/D16 tumor xenografts, 4-week-old male (NOD.CB17-Prkdcscid/ IcrCrlBltw) mice purchased from BioLASCO Taiwan Co., Ltd. (Taipei, Taiwan) were subcutaneously injected with $5 \times 10^{6}$ cells $(100 \mu 1$ in serum-free DMEM) plus $100 \mu 1$ Matrigel (354234; BD Biosciences). Animals were fed a low folic acid diet [AIN-93M folate deficient diet w/1\% succinylsulfathiazole from TestDiet (St. Louis, MO, USA)], and then randomly divided into 3 groups consisting of 4 animals each (PBS, DOC and PEM groups). Thirty days after tumors has been established, the mice were injected with DOC $(7.5 \mathrm{mg} / \mathrm{kg})$, PEM $(100 \mathrm{mg} / \mathrm{kg})$ and PBS at day-30 and -37 intraperitoneally. The tumor sizes were assessed every 3 days following drug injection and tumor volume was calculated by the following formula: $0.5 \mathrm{x}$ larger diameter $(\mathrm{mm}) \mathrm{x}$ small diameter $^{2}(\mathrm{~mm})$.

Statistical analysis. All values are presented as the mean \pm SD. Data were compared among groups using a t-test and $p<0.05$ was considered to indicate a statistically significant result.

\section{Results}

DOC-selected multi-drug resistant 4549 cells have high PEM sensitivity. According to an MTT sensitivity assay, the established A549/D16 and A549/D32 sublines revealed their drug sensitivities (Table I) in terms of $\mathrm{IC}_{50}$ (inhibition concentration) values compared with the parental A549 cells. The A549/D16 and A549/D32 sublines revealed a 109.1- and 286.59-fold resistance to DOC, respectively, when compared with the parental cells. Both sublines also exhibited cross-resistance to VCR and DXR. To investigate whether DOC resistance may result in cross-resistance to antimetabolic chemotherapy, the sensitivities to PEM and methotrexate (MTX) were also determined. Notably, only minimal resistances were detected with MTX treatment. For example; the A549/D16 and A549/D32 had a 1.8- and 2.0-fold resistance to MTX, respectively. Moreover, the A549/D16 and A549/D32 sublines had a 0.31- and 0.29-fold resistance to PEM, respectively, indicating DOC-resistant A549 sublines have higher PEM sensitivity.

PEM treatment decreases the growth of DOC-resistant A549 sublines according to a clonogenic survival assay. The DOC-resistant A549 sublines exhibited a significant sensitivity to PEM. To further characterize the cytotoxic effects of DOC and PEM to A549 and DOC-resistant sublines, we applied a clonogenic assay to determine the ability of these cells to clonally expand and create colonies. After $48 \mathrm{~h}$ of DOC exposure, the cells were cultured for 10 days and the colonies were photographed (Fig. 1A). The survival percentage was calculated as shown in Fig. 1B. None of the A549 cells survived under $16 \mathrm{nM}$ of DOC treatment, but $>80 \%$ of A549/D16 and A549/D32 cells were still able to proliferate; this confirms the DOC resistance of these sublines. When the cells were exposed to PEM for $96 \mathrm{~h}$ and maintained in fresh medium for 10 days (Fig. 1C), 33\% of A549 cells survived at the PEM concentration of $600 \mathrm{nM}$. In contrast, only 5 and 4\% of A549/D16 and A549/D32 cells remained, respectively (Fig. 1D). According to these data, we concluded that DOC-resistant A549 cells have a high sensitivity to PEM toxicity in vitro.

Downregulation of TS in the de novo DNA synthesis pathway in DOC-resistant A549 cells contributes to high PEM sensitivity. PEM is a multi-target folate synthesis inhibitor (9) and analysis has shown that high TS expression results in PEM 
$\mathbf{A}$
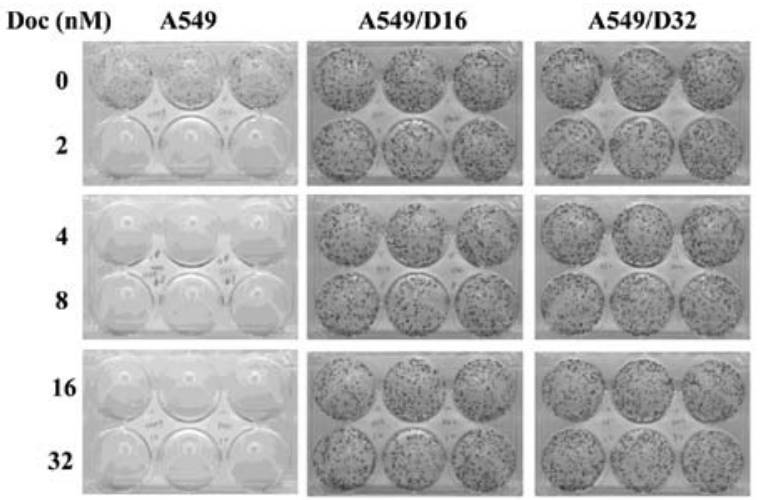

C PEM $(\mu \mathrm{M}) \quad$ A549
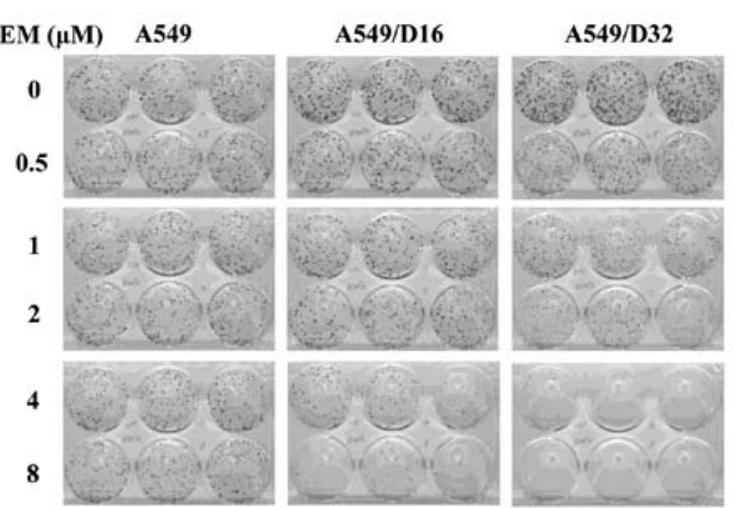

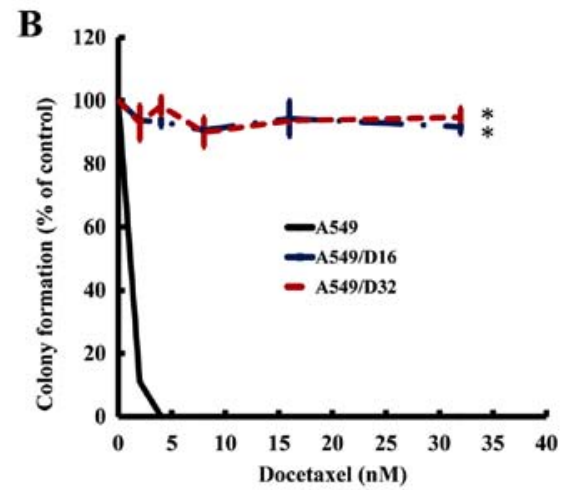

D

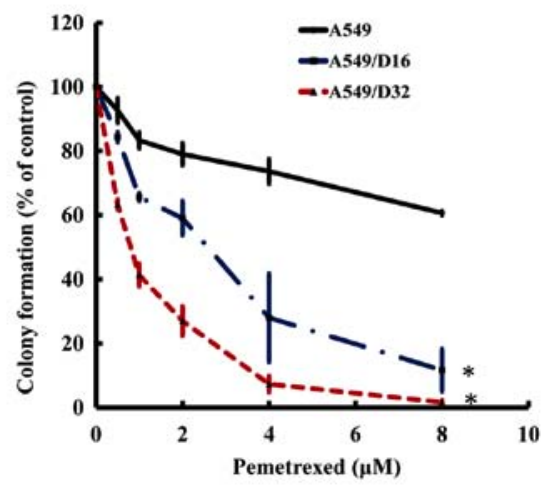

Figure 1. Evaluation of the drug sensitivities of A549 and DOC-resistant sublines by clonogenic assay. The A549 cells and sublines were seeded into 6-well plates and incubated for $24 \mathrm{~h}$. Then, the cells were treated with respective doses of DOC for $48 \mathrm{~h}$ or PEM for $96 \mathrm{~h}$, and replaced with fresh DMEM with 10\% FBS. The cells were then cultured for 10 days for colony formation. (A and C) The colonies formed were fixed and stained with 20\% Giemsa and photographed. (B and D) Survival fractions were calculated by normalization to the appropriate control groups. DOC, docetaxel; PEM; pemetrexed.
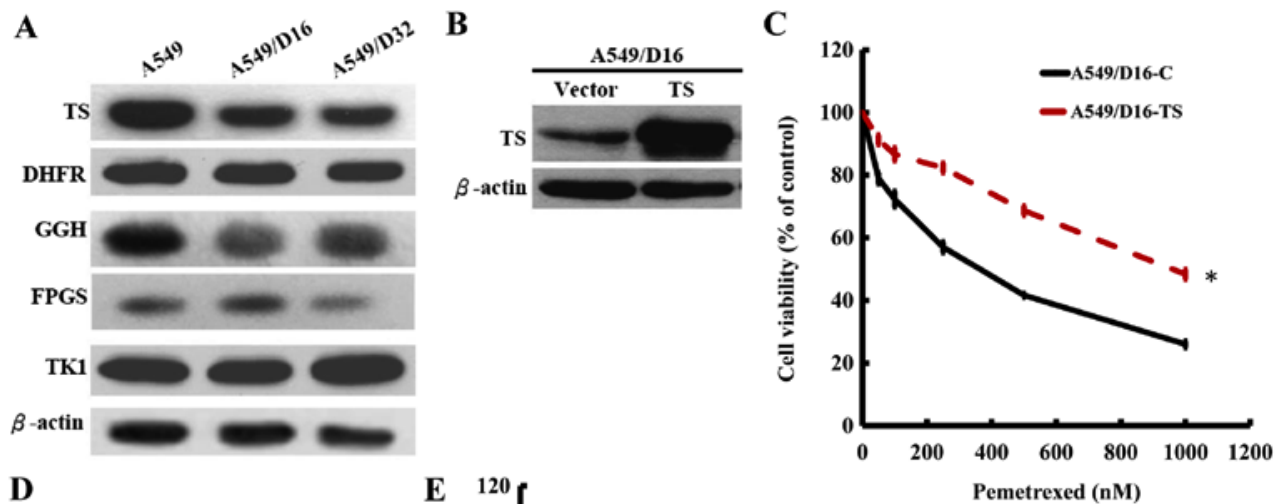

D

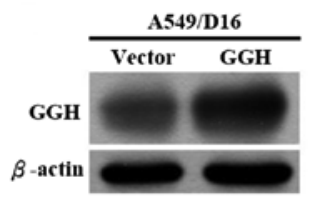

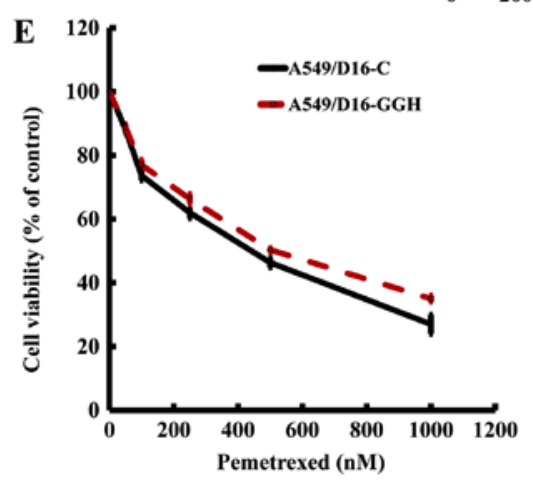

Figure 2. Characterization of the effects of TS and GGH expression on PEM sensitivities. The expression levels of folate synthesis enzymes were examined by western blot analysis in DOC-resistant A549 sublines. The enzymes of TS and DHFR are associated with de novo synthesis, and TK1 is associated with the salvage pathway of DNA synthesis. FPGS and GGH are poly/mono-glutamylation counteracting enzymes. Anti- $\beta$-actin was used as a control for loading. Representative data are shown as follows: (A) overexpression of TS in A549/D16 was detected using (B) western blotting with PEM sensitivities determined by (C) MTT assay. Similar procedures were performed with (D) GGH overexpression and the (E) effect on PEM sensitivity. TS, thymidylate synthase; GGH, $\gamma$-glutamyl hydrolase; PEM, pemetrexed; DOC, docetaxel; DHFR, dihydrofolate reductase; FPGS, folylpolyglutamate synthase. 


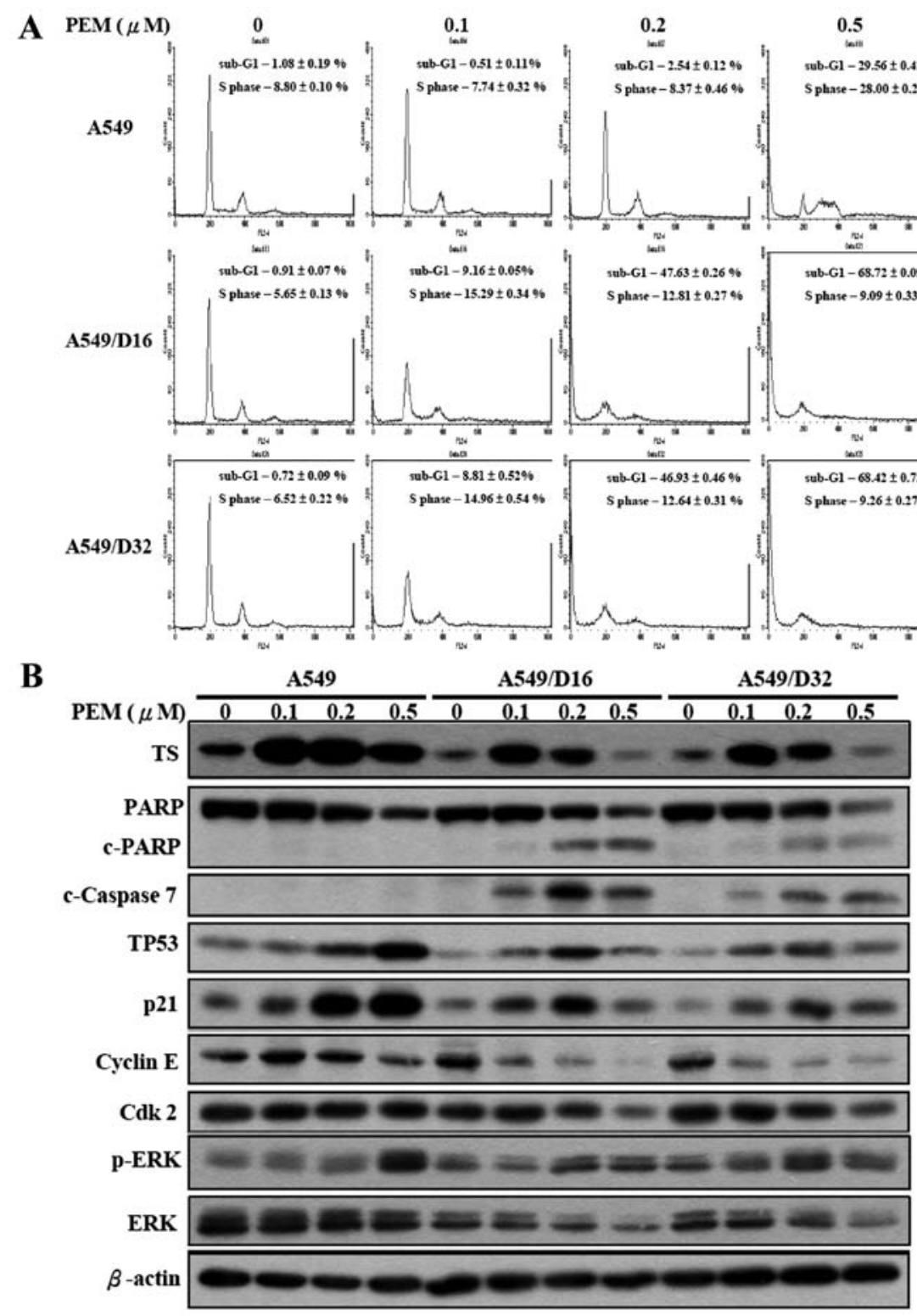

Figure 3. PEM inhibits the growth of DOC-resistant A549 cells in vitro. Effects of PEM treatment on lung cancer cells were analyzed using propidium iodide staining. (A) The sub-G1 ratios in A549 and A549/D16 cells treated with PEM (0-110 nM) for $96 \mathrm{~h}$ were examined using flow cytometry. The distribution and percentage of cells in the sub-G1 and S phases of the cell cycle are indicated. Data are expressed as the means \pm SD, and were obtained from at least 3 independent experiments. (B) The PEM-regulated proteins were further examined by western blot analysis. PEM; pemetrexed; DOC, docetaxel.

resistance $(13,16)$. Therefore, it would be logical to assume that high sensitivity to PEM may result from folate synthesis deficiency in DOC-resistant A549 cells. The enzymes of TS and DHFR are associated with de novo synthesis and TK1 is associated with the salvage pathway of DNA synthesis (17). The results from the western blot analysis revealed that only the TS protein level was significantly decreased in the DOC-resistant A549 sublines, but not the DHFR and TK1 protein levels when compared with the parental A549 cells (Fig. 2A). PEM is polyglutamated to active pentaglutamide intracellularly by a reaction catalyzed by folylpolyglutamate synthase (FPGS). The enzyme counteracting antifolate polyglutamylation is $\gamma$-glutamyl hydrolase (GGH) (9). The levels of GGH were also decreased in both sublines with slightly decreased FPGS levels observed. The data indicated that only TS in de novo DNA synthesis was downregulated in DOC-resistant A549 cells and that the low level of GGH may also contribute to PEM sensitivity by insufficiently removing polyglutamylated PEM. Therefore, we subcloned TS and GGH genes and constructed a lentiviral expression system for overexpression. To compare the PEM sensitivities, the TS-overexpressed A549/D16 and vector-control A549/D16 cells were treated with respective doses of PEM and analyzed by MTT assay. When TS was overexpressed in A549/D16 cells (Fig. 2B), the PEM sensitivity was significantly decreased (Fig. 2C). We also investigated the overexpression effect of GGH (Fig 2D) on PEM sensitivity, but insignificant results were obtained (Fig. 2E). These data demonstrated that downregulated TS is the major marker related to DOC resistance in A549 cells.

Lower concentration of PEM induces apoptotic death in vitro and upregulation of TS, TP53, p21 and phosphorylated ERK in DOC-resistant A549 cells. We further investigated the cell cycle distribution of A549 and A549/D16 cells under low 

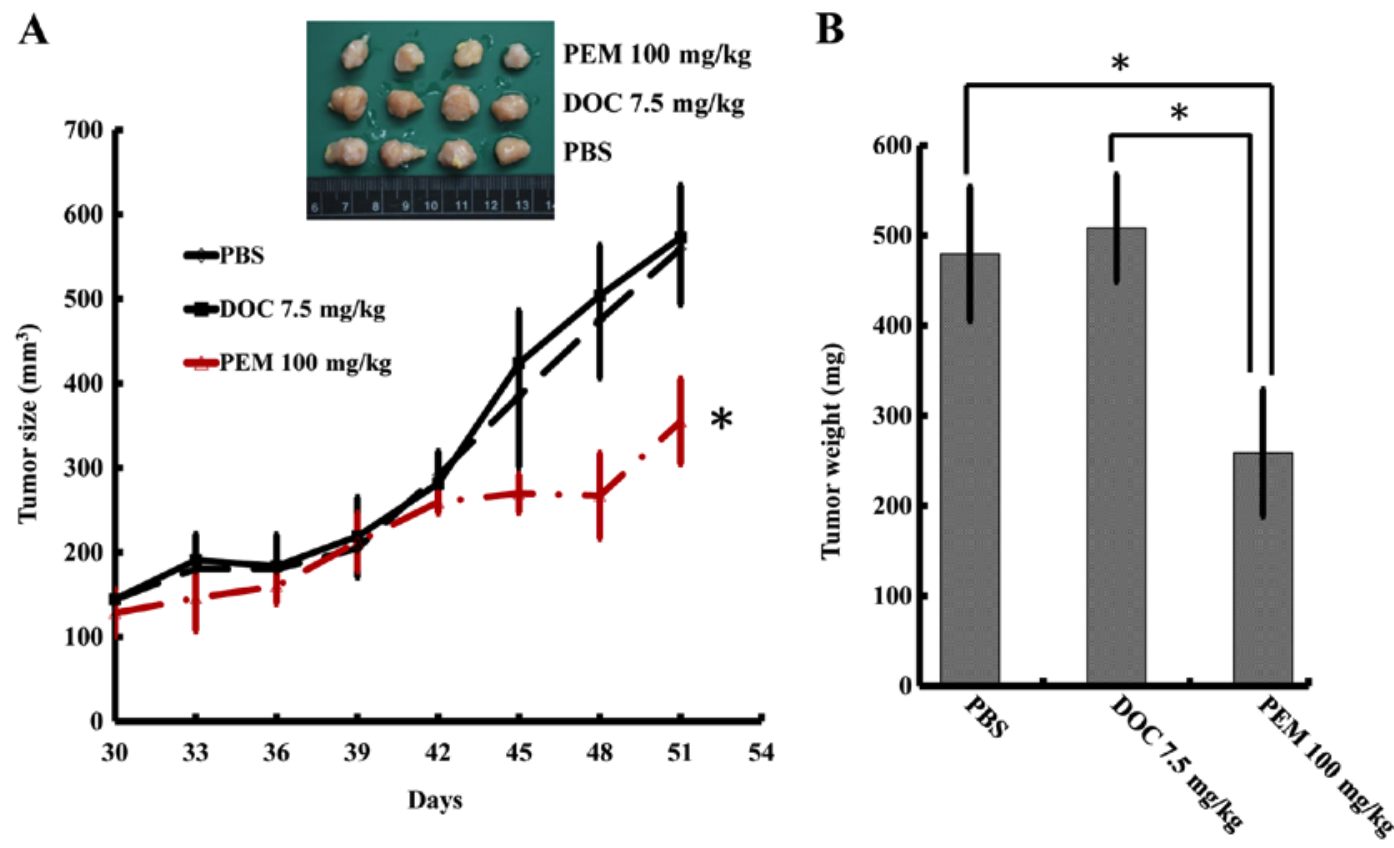

Figure 4. Inhibition of the DOC resistant A549/D16 cells by PEM in vivo. To establish A549/D16 tumor xenografts, mice were subcutaneously injected with A549/D16 cells plus Matrigel. Animals were then randomly divided into 3 groups consisting of 4 animals each (PBS, DOC and PEM groups). Thirty days after the tumors had been established, mice were injected with DOC $(7.5 \mathrm{mg} / \mathrm{kg})$, PEM $(100 \mathrm{mg} / \mathrm{kg})$ and PBS at day 30 and 37 intraperitoneally. (A) Tumor sizes were measured every 3 days following drug injection and the tumor volume was calculated. The total tumor weights from each group were also measured and listed in B. DOC, docetaxel; PEM, pemetrexed.

dosages of PEM treatment by flow cytometry. No significant effects were observed with A549 cells. Conversely, the subG1 fraction of A549/D16 cells increased with PEM concentration $(0.1 \mu \mathrm{M}, 9 \%)$, reaching a maximum level of $47 \%$ at $0.2 \mu \mathrm{M}$ of PEM treatment. Similar results were also found in A549/D32 cells (Fig. 3A). In order to delineate the molecules that were affected by PEM treatment in A549 and DOC-resistant sublines, we analyzed TS, TP53, p21 and ERK/p-ERK as previously reported (16), as well as cleaved PARP (c-PARP), caspase-7, cyclin E and Cdk2 by western blotting (Fig. 3B). Low expression levels of TS in DOC-resistant sublines were increased at $0.1 \mu \mathrm{M}$ of PEM treatment; in addition, the c-PARP and c-caspase-7 levels were also increased in correlation with apoptosis induction. Cyclin E and Cdk2 levels gradually decreased indicating that the $\mathrm{S}$ phase was arrested by PEM. TP53 and p21 were correspondingly induced, which also demonstrated that DOC-selected A549 sublines maintain TP53 and p21 activities but can be activated by lower doses of PEM when compared with parental cells. The levels of phosphorylated ERK (p-ERK) were increased in DOC-resistant sublines, suggesting that ERK phosphorylation still plays a role in PEM-induced cell death. This result is similar to the findings of previous studies $(16,18)$.

PEM inhibits the tumor growth of DOC-resistant A549 cells in vivo. To determine whether DOC resistance may be overcome by PEM in vivo, we inoculated the A549/D16 cells into mice subcutaneously. The mice were fed an AIN-93M folate deficient diet $\mathrm{w} / 1 \%$ succinylsulfathiazole (catalog no. 1812813; TestDiet) to increase PEM sensitivity (19). The PBS group served as tumor growth controls and the DOC group served as evidence for DOC resistance. Since DOC presents significant toxicity to animals, the experiments were terminated after
51 days. The tumors treated with PEM exhibited significant growth inhibition, according to size measurements (Fig. 4A). The total weights of the tumors were assessed and are listed in Fig. 4B, which revealed that the tumors in the group of PEM-treated mice significantly weighed less than the tumors in the groups treated with PBS or DOC.

DOC induces wild-type TP53 and downregulates TS. Previously, we reported that DOC induces TP53 transcription in A549 cells (20). Therefore, we proposed that long-term exposure to DOC which downregulates the folate synthesis enzymes in A549 cells may be associated with TP53 activity. To investigate this hypothesis, several lung cancer cell lines were selected; the H460 cells had wild-type TP53, the H1299 cells had null TP53, and the H1355 and CL1-0 cells had mutated TP53. All cancer cell lines were treated with sub-lethal doses of DOC as indicated and analyzed by western blotting. The levels of MDM2 and TP53 proteins should be increased when wild-type TP53 is activated $(21,22)$. This was demonstrated in the present study when A549 and H460 cells were exposed to DOC (Fig. 5A). Neither MDM2 nor TP53 protein levels were altered in H1299, H1355 and CL1-0 cells treated with DOC. These data support our hypothesis that inducing TP53 inhibits TS expression. To determine whether the association between TP53 and TS depends on drug concentration, various doses of DOC were added to cells and analyzed by western blot analyses (Fig. 5B). Downregulated TS expressions was correlated with increased DOC concentrations. We previously established an RNAi-p53-A549 cell line clone in which TP53 was inhibited (23). The levels of TS were significantly increased (Fig. 5C) in these TP53-inhibited A549 cells. According to our data, the expression of the TS protein was regulated by wild-type TP53. 


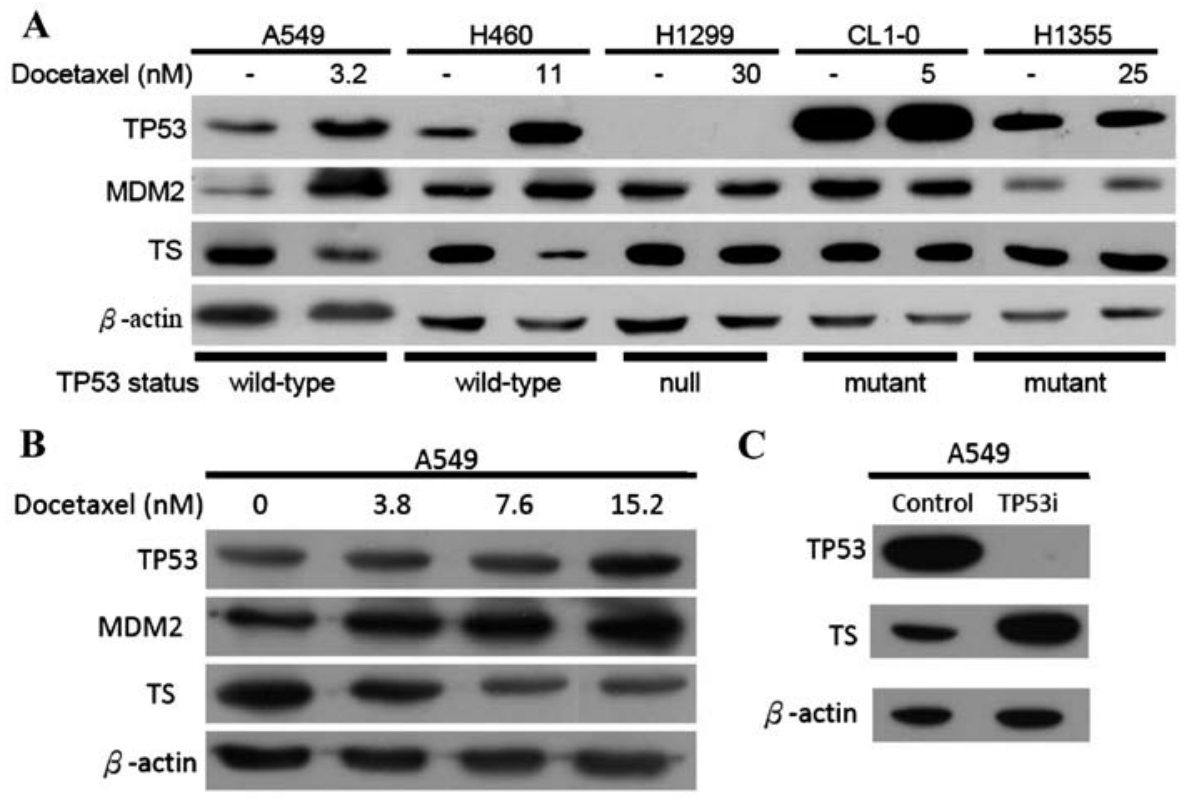

Figure 5. Association of the TP53 status and TS expression with DOC treatment. (A) The expression levels of TP53, MDM2 and TS altered by DOC treatment were investigated by western blot analysis in A549, H460, H1299, CL1-0 and H1355 cells. (B) Dose-dependence of DOC in TP53, MDM2 and TS expression were characterized by western blot analyses. (C) The results of interference by si-TS RNA in A549 cells were investigated by western blot analysis for TS expression. TS, thymidylate synthase; DOC, docetaxel.

\section{A549/Docetaxel resistance and high pemetrexed sensitivity}

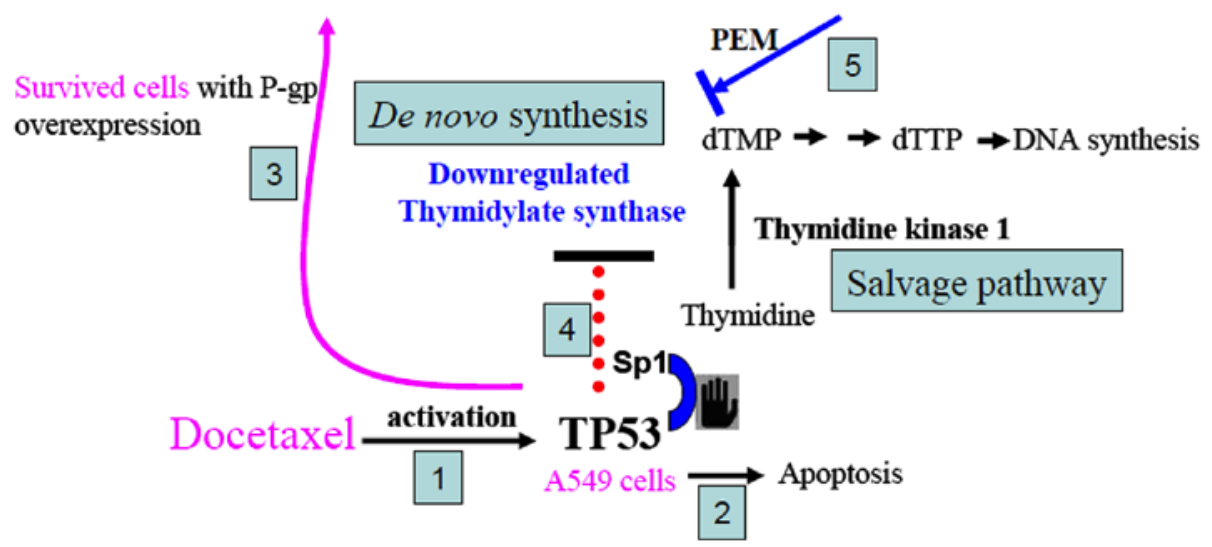

Figure 6. A proposed mechanism for TP53-mediated TS downregulation in DOC-resistant A549 cells. (1) DOC treatment activates TP53, (2) induces apoptotic death for most of the cells. (3) The surviving cells develop high P-gp expression. (4) In these DOC-resistant cells, activated TP53 occupies the Sp1 region and downregulates TS expression. (5) After DOC selection, A549 cells with downregulated TS are more sensitive to PEM than the parental A549 cells. TS, thymidylate synthase; DOC, docetaxel; P-gp, P-glycoprotein; PEM, pemetrexed.

\section{Discussion}

In 2005 in the UK, the National Institute for Health and Care Excellence (NICE) produced comprehensive guidelines on the management of patients with lung cancer. These guidelines recommended chemotherapy for NSCLC patients; DOC, gemcitabine (GEM), paclitaxel (PAX) or vinorelbine (VNB) in combination with either cisplatin (CIS) or carboplatin (CARB) were recommended as standard first-line treatments for patients with advanced locally or metastatic disease. Further guidance has been published which recommends PEM in combination with CIS as a first-line treatment for patients with non-squamous locally advanced or metastatic disease (24). In the same study, the effectiveness of first-line chemotherapy was evaluated using electronic databases (MEDLINE, EMBASE and Cochrane Library). In patients with non-squamous disease, PEM + platinum (PLAT) increased overall survival (OS) statistically and significantly compared with GEM + PLAT and DOC + PLAT increased OS statistically and significantly compared with PAX + PLAT (24). DOC is an anti-microtubule agent and PEM is an antifolate agent; therefore, they target different cellular proteins for cancer treatment (25). In addition to the enormous efforts being made to assist clinicians in making decisions regarding how to treat patients of advanced NSCLC with various anticancer drugs, we endeavored to define the influencing factors that may relieve DOC-resistance using another anticancer drug as second-line chemotherapy. 
We established DOC-resistant A549 sublines and defined their sensitivities with various anticancer drugs (DOC, VCR, DXR, MTX and PEM). Notably, the DOC-resistant A549 sublines not only avoided cross-resistance to PEM, in contrast, those sublines were also more sensitive to PEM than parental A549 cells. According to our previous study (15), A549/D16 and A549/D32 cells were resistant to DOC due to overexpression of P-gp. Therefore, we concluded that P-gp overexpression does not affect PEM sensitivity. Our data also demonstrated that only TS, neither DHFR nor TK1, was downregulated in DOC-resistant sublines. Overexpressing TS decreased PEM cytotoxicity in DOC-resistant cells. Furthermore, although a low GGH expression level indicates less ability to counteract the multi-targeted action of PEM generated by FPGS-mediated polyglutamylation, overexpression of GGH in DOC-resistant A549 cells has little effect on decreasing PEM cytotoxicity. Higher PEM sensitivity in DOC-resistant sublines can be attenuated by overexpressing the TS gene. This indicates that low TS expression is a major factor in PEM sensitivity. Therefore, finding a way to decrease TS expression in NSCLC before PEM application may be worthy of more investigation to improve the efficacy of PEM.

It has been demonstrated that wild-type TP53 inhibits mouse TS promoter activity by $>95 \%$. In contrast, an expression plasmid that encodes a mutant form of TP53 has little effect on mouse TS promoter activity (26). To determine whether human TS expression is associated with TP53 status, we examined several human lung cancer cell lines under DOC treatment and tested to see whether TS expression could be regulated. Our data demonstrated that the level of TS protein was significantly decreased only in wild-type TP53 containing human lung cells (A549 and H460) that had been treated by DOC in a dose-dependent manner. Conversely, TS and TP53 levels were reported in 84 breast tumor samples that were examined using immunohistochemistry (27). Another study revealed that wild-type TP53 status was associated with low protein expression of TS in human colon carcinoma cell lines LoVo 92 (wild-type TP53) and WiDr T (mutant TP53) (28). Previously, we reported that DOC significantly induced human wild-type TP53 transcription (20,23), however induced wild-type TP53 associated with a TS decrease in human cancer cells has yet to be demonstrated. Another interesting finding was that mutant TP53 could not be activated by DOC, which was demonstrated by the constant levels of MDM2 expression in H1355 and CL1-0 cancer cells containing mutant TP53 (Fig. 5A). Therefore, the status of TP53 which altered the expression of TS was not only found in our lung cancer model, it was also found in breast and colon cancers, as reported by other researchers. Although the mechanism involving DOC-induced TP53 suppression of TS expression was not examined in the present study, we proposed a possible link to TS inhibition. Previously, the upstream of the essential promoter region of human TS was isolated. The promoter region of the human TS gene does not contain any DNA motifs that are typical of eukaryotic promoters, such as a TATA box, a CAAT box, or a typical GC box. Notably, a CACCC box and an Sp1-binding motif were identified revealing that the Sp1-binding motif acts as the core sequence of the promoter to maintain the basic promoter activity of genes for human TS (29). Notably, it has been demonstrated that oxaliplatin treatment induces enrichment of TP53 at the deoxyuridine triphosphate nucleotidohydrolase (dUTPase) promoter with a concomitant decrease in $\mathrm{Sp} 1$ and a transcriptional suppression of dUTPase (30). The 5 ' region of the human dUTPase gene has been identified as a binding site for $\mathrm{Spl}$ and it has been suggested that TP53 acts on the region of the promoter containing the Sp1 sites, which may prevent activation of the dUTPase gene by Sp1. Therefore, we provide a plausible mechanism to explain why TS expression was inhibited in DOC-resistant A549 cells (Fig. 6). Our previously reported data revealed that the initial response of cancer cells to DOC treatment involved wild-type TP53 (23). DOC treatment activated TP53 and induced apoptotic death for most cells. The surviving cells developed high P-gp expression. In these DOC-resistant cells, activated TP53 occupied the Sp1 region and downregulated TS expression. The salvage pathway of TK1 may provide essential dTMP for TS-suppressed cells to continuously proliferate as these cells are gradually selected by higher doses of DOC in vitro. Therefore, by DOC selection, we demonstrated that A549 cells with downregulated TS were more sensitive to PEM than parental A549 cells.

Our data revealed that application of DOC in wild-type TP53 NSCLC as first-line chemotherapy may be a better choice than VNB or VCR, which have similar microtubule destabilization mechanisms. Indeed, mutations of the TP53 gene occur in $\sim 50 \%$ of NSCLC $(31,32)$. Determining TP53 status in NSCLC may be of great value in the choice of firstline chemotherapy and management of resistance.

Recent advances in chemotherapy are welcome, but their effects remain small for patients with NSCLC. In the present study, we revealed that the chemotherapeutic drug DOC induced wild-type, but not mutant TP53 and inhibited TS expression. The de novo nucleic acid synthesis pathway was downregulated in DOC-resistant lung cancer cells, resulting in high PEM sensitivity.

We wondered what the optimal chemotherapy treatment was for recurrent NSCLC patients who have previously received taxane-based chemotherapy. Ideally, we proposed that for lung cancer with wild-type TP53, DOC should be used as the first-line anticancer drug. When DOC-resistance develops, PEM could be used as the second-line treatment when the TS expression level is examined accordingly. Our findings revealed that the sequence of chemotherapy treatment can be optimized with DOC followed by PEM in wild-type TP53 NSCLC. This sequence is well suited for extended treatment intervals due to such properties as absence of cumulative toxicity, non-cross-resistance, positive benefits on the quality of life, and convenient scheduling. Undoubtedly, PEM may continue to be used extensively in patients with NSCLC.

\section{Acknowledgements}

The present study was supported by the Ditmanson Medical Foundation Chia-Yi Christian Hospital (grant no. R105-023) and a grant to M.-F.W. (no. CSH-2014-C-007).

\section{References}

1. Torre LA, Bray F, Siegel RL, Ferlay J, Lortet-Tieulent J and Jemal A: Global cancer statistics, 2012. CA Cancer J Clin 65: 87-108, 2015. 
2. Roengvoraphoj M, Tsongalis GJ, Dragnev KH and Rigas JR Epidermal growth factor receptor tyrosine kinase inhibitors as initial therapy for non-small cell lung cancer: Focus on epidermal growth factor receptor mutation testing and mutation-positive patients. Cancer Treat Rev 39: 839-850, 2013.

3. Alamgeer M, Ganju V and Watkins DN: Novel therapeutic targets in non-small cell lung cancer. Curr Opin Pharmacol 13: 394-401, 2013.

4. Fitzpatrick FA and Wheeler R: The immunopharmacology of paclitaxel (Taxol), docetaxel (Taxotere), and related agents. Int Immunopharmacol 3: 1699-1714, 2003.

5. Horwitz SB: Mechanism of action of taxol. Trends Pharmacol Sci 13: 134-136, 1992.

6. Leonard GD, Fojo T and Bates SE: The role of ABC transporters in clinical practice. Oncologist 8: 411-424, 2003.

7. Fojo AT and Menefee M: Microtubule targeting agents: Basic mechanisms of multidrug resistance (MDR). Semin Oncol 32 (Suppl 7): S3-S8, 2005.

8. Baldwin CM and Perry CM: Pemetrexed: A review of its use in the management of advanced non-squamous non-small cell lung cancer. Drugs 69: 2279-2302, 2009.

9. Shih C, Habeck LL, Mendelsohn LG, Chen VJ and Schultz RM: Multiple folate enzyme inhibition: Mechanism of a novel pyrrolopyrimidine-based antifolate LY231514 (MTA). Adv Enzyme Regul 38: 135-152, 1998.

10. Goldman ID and Zhao R: Molecular, biochemical, and cellular pharmacology of pemetrexed. Semin Oncol 29 (Suppl 18): S3-S17, 2002.

11. Zhang D, Ochi N, Takigawa N, Tanimoto Y, Chen Y, Ichihara E, Hotta K, Tabata M, Tanimoto M and Kiura K: Establishment of pemetrexed-resistant non-small cell lung cancer cell lines. Cancer Lett 309: 228-235, 2011

12. Chen CY, Chang YL, Shih JY, Lin JW, Chen KY, Yang CH, Yu CJ and Yang PC: Thymidylate synthase and dihydrofolate reductase expression in non-small cell lung carcinoma: The association with treatment efficacy of pemetrexed. Lung Cancer 74 132-138, 2011

13. Liu Y, Yin TJ, Zhou R, Zhou S, Fan L and Zhang RG: Expression of thymidylate synthase predicts clinical outcomes of pemetrexed-containing chemotherapy for non-small-cell lung cancer: A systemic review and meta-analysis. Cancer Chemother Pharmacol 72: 1125-1132, 2013.

14. Sigmond J, Backus HH, Wouters D, Temmink OH, Jansen G and Peters GJ: Induction of resistance to the multitargeted antifolate Pemetrexed (ALIMTA) in WiDr human colon cancer cells is associated with thymidylate synthase overexpression. Biochem Pharmacol 66: 431-438, 2003.

15. Chiu LY, Ko JL, Lee YJ, Yang TY, Tee YT and Sheu GT: L-type calcium channel blockers reverse docetaxel and vincristineinduced multidrug resistance independent of $A B C B 1$ expression in human lung cancer cell lines. Toxicol Lett 192: 408-418, 2010.

16. Chiu LY, Hsin IL, Yang TY, Sung WW, Chi JY, Chang JT, Ko JL and Sheu GT: The ERK-ZEB1 pathway mediates epithelialmesenchymal transition in pemetrexed resistant lung cancer cells with suppression by vinca alkaloids. Oncogene 36: 242-253, 2017.

17. Shintani M, Urano M, Takakuwa Y, Kuroda M and Kamoshida S: Immunohistochemical characterization of pyrimidine synthetic enzymes, thymidine kinase- 1 and thymidylate synthase, in various types of cancer. Oncol Rep 23: 1345-1350, 2010.

18. Yang TY, Chang GC, Chen KC, Hung HW, Hsu KH, Sheu GT and Hsu SL: Sustained activation of ERK and Cdk2/cyclin-A signaling pathway by pemetrexed leading to S-phase arrest and apoptosis in human non-small cell lung cancer A549 cells. Eur J Pharmacol 663: 17-26, 2011.
19. van der Wilt CL, Backus HH, Smid K, Comijn L, Veerman G, Wouters D, Voorn DA, Priest DG, Bunni MA, Mitchell F, et al: Modulation of both endogenous folates and thymidine enhance the therapeutic efficacy of thymidylate synthase inhibitors. Cancer Res 61: 3675-3681, 2001 .

20. Chuang JC, Sheu GT, Wang PC, Liao FT, Liu WS, Huang CF, Tseng MH and Wu MF: Docetaxel and 5-fluorouracil induce human p53 tumor suppressor gene transcription via a short sequence at core promoter element. Toxicol In Vitro 26: 678-685, 2012.

21. Barak Y, Juven T, Haffner R and Oren M: mdm2 expression is induced by wild type p53 activity. EMBO J 12: 461-468, 1993.

22. Wu X, Bayle JH, Olson D and Levine AJ: The p53-mdm-2 autoregulatory feedback loop. Genes Dev 7: 1126-1132, 1993.

23. Chang JT, Chang GC, Ko JL, Liao HY, Liu HJ, Chen CC, Su JM, Lee $\mathrm{H}$ and Sheu GT: Induction of tubulin by docetaxel is associated with p53 status in human non small cell lung cancer cell lines. Int J Cancer 118: 317-325, 2006.

24. Brown T, Pilkington G, Bagust A, Boland A, Oyee J, Tudur-Smith C, Blundell M, Lai M, Martin Saborido C, Greenhalgh J, et al: Clinical effectiveness and cost-effectiveness of first-line chemotherapy for adult patients with locally advanced or metastatic non-small cell lung cancer: A systematic review and economic evaluation. Health Technol Assess 17: 1-278, 2013.

25. Yang TY, Chang GC, Chen KC, Hung HW, Hsu KH, Wu CH, Sheu GT and Hsu SL: Pemetrexed induces both intrinsic and extrinsic apoptosis through ataxia telangiectasia mutated/ p53-dependent and -independent signaling pathways. Mol Carcinog 52: 183-194, 2013.

26. Lee Y, Chen Y, Chang LS and Johnson LF: Inhibition of mouse thymidylate synthase promoter activity by the wild-type p53 tumor suppressor protein. Exp Cell Res 234: 270-276, 1997.

27. Calascibetta A, Martorana A, Cabibi D, Aragona F and Sanguedolce R: Relationship between thymidylate synthase and p53 and response to FEC versus taxane adjuvant chemotherapy for breast carcinoma. J Chemother 23: 354-357, 2011.

28. Giovannetti E, Backus HH, Wouters D, Ferreira CG, van Houten VM, Brakenhoff RH, Poupon MF, Azzarello A, Pinedo HM and Peters GJ: Changes in the status of p53 affect drug sensitivity to thymidylate synthase (TS) inhibitors by altering TS levels. Br J Cancer 96: 769-775, 2007.

29. Horie $\mathrm{N}$ and Takeishi K: Identification of functional elements in the promoter region of the human gene for thymidylate synthase and nuclear factors that regulate the expression of the gene. J Biol Chem 272: 18375-18381, 1997.

30. Wilson PM, Fazzone W, LaBonte MJ, Lenz HJ and Ladner RD: Regulation of human dUTPase gene expression and p53-mediated transcriptional repression in response to oxaliplatin-induced DNA damage. Nucleic Acids Res 37: 78-95, 2009.

31. Bodner SM, Minna JD, Jensen SM, D'Amico D, Carbone D, Mitsudomi T, Fedorko J, Buchhagen DL, Nau MM, Gazdar AF, et al: Expression of mutant $\mathrm{p} 53$ proteins in lung cancer correlates with the class of p53 gene mutation. Oncogene 7: 743-749, 1992.

32. Takahashi T, Nau MM, Chiba I, Birrer MJ, Rosenberg RK, Vinocour M, Levitt M, Pass H, Gazdar AF and Minna JD: p53: A frequent target for genetic abnormalities in lung cancer. Science 246: 491-494, 1989. 